\title{
Solving NP-complete Problems by Spiking Neural P Systems with Budding Rules
}

\author{
Tseren-Onolt Ishdorj ${ }^{1}$, Alberto Leporati ${ }^{2}$, Linqiang Pan $^{3,4}$, Jun Wang $^{3}$ \\ 1 Computational Biomodelling Laboratory \\ Åbo Akademi University \\ Department of Information Technologies \\ 20520 Turku, Finland \\ tishdorj@abo.fi \\ 2 Università degli Studi di Milano - Bicocca \\ Dipartimento di Informatica, Sistemistica e Comunicazione \\ Viale Sarca 336/14, 20126 Milano, Italy \\ alberto.leporati@unimib.it \\ ${ }^{3}$ Key Laboratory of Image Processing and Intelligent Control \\ Department of Control Science and Engineering \\ Huazhong University of Science and Technology \\ Wuhan 430074, Hubei, People's Republic of China \\ junwangjf@gmail.com, lqpan@mail.hust.edu.cn \\ ${ }^{4}$ Research Group on Natural Computing \\ Department of CS and AI, University of Sevilla \\ Avda Reina Mercedes s/n, 41012 Sevilla, Spain
}

Summary. Inspired by the growth of dendritic trees in biological neurons, we introduce spiking neural $\mathrm{P}$ systems with budding rules. By applying these rules in a maximally parallel way, a spiking neural $\mathrm{P}$ system can exponentially increase the size of its synapse graph in a polynomial number of computation steps. Such a possibility can be exploited to efficiently solve computationally difficult problems in deterministic polynomial time, as it is shown in this paper for the NP-complete decision problem SAT.

\section{Introduction}

Spiking neural P systems (SN P systems, for short) have been introduced in [5] as a new class of distributed and parallel computing devices, inspired by the neurophysiological behavior of neurons sending electrical impulses (spikes) along axons to other neurons. SN P systems can also be viewed as an evolution of $\mathrm{P}$ systems $[19,16]$ corresponding to a shift from cell-like to neural-like architectures. We recall that this biological background has already led to several models in the area of neural computation, e.g., see $[13,14,4]$. 
In SN P systems the cells (also called neurons) are placed in the nodes of a directed graph, called the synapse graph. The contents of each neuron consist of a number of copies of a single object type, called the spike. Every cell may also contain a number of firing and forgetting rules. Firing rules allow a neuron to send information to other neurons in the form of electrical impulses (also called spikes) which are accumulated at the target cells. The applicability of each rule is determined by checking the contents of the neuron against a regular set associated with the rule. In each time unit, if a neuron can use some of its rules then one of such rules must be used. The rule to be applied is nondeterministically chosen. Thus, the rules are used in a sequential manner in each neuron, but neurons function in parallel with each other. Observe that, as usually happens in membrane computing, a global clock is assumed, marking the time for the whole system, hence the functioning of the system is synchronized. When a cell sends out spikes it becomes "closed" (inactive) for a specified period of time, that reflects the refractory period of biological neurons. During this period, the neuron does not accept new inputs and cannot "fire" (that is, emit spikes). Another important feature of biological neurons is that the length of the axon may cause a time delay before a spike reaches its target. In $\mathrm{SN}$ P systems this delay is modeled by associating a delay parameter to each rule which occurs in the system. If no firing rule can be applied in a neuron, there may be the possibility to apply a forgetting rule, that removes from the neuron a predefined number of spikes.

The computational efficiency of SN P systems has been recently investigated in a series of works $[2,6,9,11,10]$. In [12] it has been proved that a deterministic SN $\mathrm{P}$ system of polynomial size cannot solve an NP-complete problem in a polynomial time, unless $\mathbf{P}=\mathbf{N P}$. Hence, under the assumption that $\mathbf{P} \neq \mathbf{N P}$, efficient solutions to NP-complete problems cannot be obtained without introducing features which enhance the efficiency, such as pre-computed resources, ways to exponentially grow the workspace during the computation, nondeterminism, and so on. Indeed, in the framework of SN P systems, most of the solutions to computationally hard problems exploit the power of nondeterminism $[11,10,12]$ or use pre-computed resources of exponential size $[2,6,9,7]$.

The possibility of using SN P systems to solve computationally hard problems by using some (possibly exponentially large) pre-computed resources has been first presented in [6], that contains a description of a uniform family of SN P systems with pre-computed resources of exponential size that solves all the instances of the NP-complete decision problem SAT in a polynomial time. In the present paper we complement the study exposed in [6], by describing an SN P system that first builds the necessary resources (by exponentially increasing its workspace in a polynomial time), and then uses such resources to solve the SAT problem. To this purpose, we extend the SN P systems given in [6] by introducing neuron budding rules. We show that SN P systems with budding rules can grow an exponential size synapse graph in a time which is polynomial with respect to the size of the instances of the problem we want to solve. Then, the systems themselves can be used to solve such instances. All the systems we will propose work in a deterministic way. 
The biological motivation for the mechanism that we use to expand the synapse graph of SN P systems comes from the growth of dendritic trees in biological neurons [20]. It is known that the human brain is made up of about 100 billion cells. Almost all brain cells are formed before birth. Dendrites (from the Greek, "tree") are the branched projections of a neuron. The point at which the dendrites of a cell come into contact with the dendrites of another cell is where the miracle of information transfer (communication) occurs. Brain cells can grow as many as one billion of dendrite connections - a universe of touch points. The greater the number of dendrites, the more information can be processed. Dendrites grow as a result of stimulation from and interaction with their environment. With limited stimulation there is limited growth; with no stimulation, dendrites actually retreat and disappear. The microscope photographs illustrated in Figure 1 show actual dendrite development. Dendrites begin to emerge from a single neuron (brain cell) and develop into a cluster of touch points seeking to connect with dendrites from other cells.

In the framework of SN P systems, the dendrite connection points are modelled as abstract neurons, while the branches of dendrite trees are modelled as abstract synapses. A new connection between dendrites coming from two different neuron cells is understood as a newly created synapse. In this way, new neurons and new synapses can be produced during the growth of a dendrite tree. The formal definition of neuron budding rule and its semantics will be given in Section 2 .

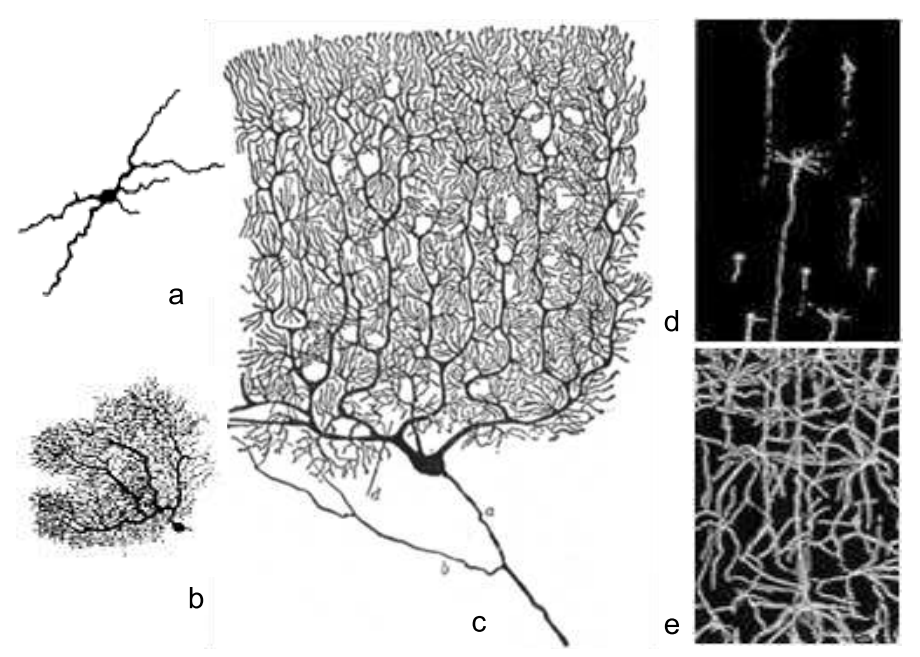

Fig. 1. A growing neuron: a. dendrites begin to emerge from a single neuron, b. dendrites developed into a cluster of touch points; c. Ramon y Cajal, Santiago. Classical drawing: Purkinje cell; d. newborn neuron dendrites, e. three months later. Photos from Tag Toys $[20]$ 


\section{SN P systems with budding rules}

A spiking neural $P$ system with budding rules, of initial degree $m \geq 1$, is a construct of the form

$$
\Pi=(O, \Sigma, H, \text { syn }, R, \text { in }, \text { out }),
$$

where:

1. $O=\{a\}$ is the singleton alphabet ( $a$ is called spike);

2. $\Sigma=\left\{\sigma_{1}, \sigma_{2}, \ldots, \sigma_{m}\right\}$ is a finite set of initial neurons;

3. $H$ is a finite set of labels for neurons;

4. syn $\subseteq H \times H$ is a finite set of synapses, with $(i, i) \notin$ syn for $i \in H$;

5. $R$ is a finite set of developmental rules, of the following forms:

(1) neuron budding rule $x[]_{i} \rightarrow y[]_{j}$, where $x \in\{(k, i),(i, k), \lambda\}, y \in$ $\{(i, j),(j, i), \lambda\}, i, j, k \in H, i \neq k, i \neq j$;

(2) extended firing (also called spiking) rule $\left[E / a^{c} \rightarrow a^{p} ; d\right]_{i}$, where $i \in H, E$ is a regular expression over $a$, and $c \geq 1, p \geq 0, d \geq 0$ are integer numbers, with the restriction $c \geq p$.

6. in, out $\in H$ indicate the input and the output neurons of $\Pi$.

Note that the definition of SN P systems with budding rules is slightly different from the usual definition of SN P systems given in the literature, where the neurons that occur in the system are explicitly listed as $\sigma_{i}=\left(n_{i}, R_{i}\right), 1 \leq i \leq m$, where $R_{i}$ is the set of rules associated with neuron $\sigma_{i}$, and $n_{i}$ is the number of spikes it contains in the initial configuration of the system. First of all, only the structure of the system is given in our definition; the presence of spikes (if any) in the initial configuration is specified at the beginning of each computation. Further, $i$ is considered as the label of neuron $\sigma_{i}$. In SN P systems with budding rules it is possible to create new neurons in the course of a computation; hence the system may contain, in a given configuration, several neurons that are labelled with the same element of $H$. Nonetheless, with a slight abuse of notation in what follows we will refer to any neuron having the label $i \in H$ by calling it $\sigma_{i}$.

Considering the budding rule $x[]_{i} \rightarrow y[]_{j}$, its left hand side describes the neuron $\sigma_{i}$ with a synapse $x$ connected with one of its neighbouring neurons, to which the rule is supposed to be applied. The right hand side describes the result of the rule application, that is, the newly created neuron $\sigma_{j}$ and synapse $y$. Note that for the sake of simplicity, in the rule notation we omit to repeat the contents of the left hand side of the rule in the right hand side. We say that the rule is restricted because only one neighbouring neuron is considered in each side of the rule.

A budding rule can be applied only if the neighbourhood of the associated neuron is exactly as described in the left hand side of the rule, in other words, $x=X$ where $X$ is the current set of synapses of neuron $\sigma_{i}$. As a result of the rule application, a new neuron $\sigma_{j}$ and a synapse $y$ are established, provided that they do not already exist; if a neuron with label $j$ already exists in the system but no synapse of type $y$ exists, then only the synaptic connection $y$ between the neurons 
$\sigma_{i}$ and $\sigma_{j}$ is established; no new neuron with label $j$ is budded. We stress here that the application of budding rules does not depend on the spikes contained into the neuron. Budding rules are applied in a maximally parallel way: if the neighbourhood of neuron $\sigma_{i}$ enables several budding rules, then all these rules are applied in parallel; as a result, several new neurons and synapses are produced (which corresponds to have several branches at a touch point in the dendrite tree). Note that the way of using neuron budding rules is different with respect to the usual way in which $\mathrm{P}$ systems with active membranes use cell division or cell creation rules, where at most one of these rules can be applied inside each membrane during a computation step.

Extended firing rules are defined as usually done in SN P systems. If an extended firing rule $\left[E / a^{c} \rightarrow a^{p} ; d\right]_{i}$ has $E=a^{c}$, then we will write it in the simplified form $\left[a^{c} \rightarrow a^{p} ; d\right]_{i}$; similarly, if a rule $\left[E / a^{c} \rightarrow a^{p} ; d\right]_{i}$ has $d=0$, then we can simply write it as $\left[E / a^{c} \rightarrow a^{p}\right]_{i}$; hence, if a rule $\left[E / a^{c} \rightarrow a^{p} ; d\right]_{i}$ has $E=a^{c}$ and $d=0$, then we can write $\left[a^{c} \rightarrow a^{p}\right]_{i}$. A rule $\left[E / a^{c} \rightarrow a^{p}\right]_{i}$ with $p=0$ is written in the form $\left[E / a^{c} \rightarrow \lambda\right]_{i}$ and is called an extended forgetting rule. Rules of the types $\left[E / a^{c} \rightarrow a ; d\right]_{i}$ and $\left[a^{c} \rightarrow \lambda\right]_{i}$ are said to be standard. However, even in this case we do not require that if a forgetting rule is enabled then no firing rules are also enabled at the same time in the same neuron, as it happens in standard SN P systems.

If a neuron $\sigma_{i}$ contains $k$ spikes and $a^{k} \in L(E), k \geq c$, then the rule $\left[E / a^{c} \rightarrow\right.$ $\left.a^{p} ; d\right]_{i}$ is enabled and can be applied. This means consuming (removing) $c$ spikes (thus only $k-c$ spikes remain in neuron $\sigma_{i}$ ); the neuron is fired, and it produces $p$ spikes after $d$ time units. If $d=0$, then the spikes are emitted immediately; if $d=1$, then the spikes are emitted in the next step, etc. If the rule is used in step $t$ and $d \geq 1$, then in steps $t, t+1, t+2, \ldots, t+d-1$ the neuron is closed (this corresponds to the refractory period from neurobiology), so that it cannot receive new spikes (if a neuron has a synapse to a closed neuron and tries to send a spike along it, then that particular spike is lost). In the step $t+d$, the neuron spikes and becomes open again, so that it can receive spikes (which can be used starting with the step $t+d+1$, when the neuron can again apply rules). Once emitted from neuron $\sigma_{i}$, the $p$ spikes reach immediately all neurons $\sigma_{j}$ such that there is a synapse going from $\sigma_{i}$ to $\sigma_{j}$ and which are open, that is, the $p$ spikes are replicated and each target neuron receives $p$ spikes; as stated above, spikes sent to a closed neuron are "lost", that is, they are removed from the system. In the case of the output neuron, $p$ spikes are also sent to the environment. Of course, if neuron $\sigma_{i}$ has no synapse leaving from it, then the produced spikes are lost. If the rule is a forgetting one of the form $\left[E / a^{c} \rightarrow \lambda\right]_{i}$, then, when it is applied, $c \geq 1$ spikes are removed. When a neuron is closed, none of its rules can be used until it becomes open again.

In each time unit, if a neuron $\sigma_{i}$ can use one of its rules, then a rule from $R$ must be used. If the neighbourhood of neuron $\sigma_{i}$ enables several budding rules, then all these rules are applied in parallel. If several spiking rules are enabled in neuron $\sigma_{i}$, then only one of them is nondeterministically chosen. If both spiking 
rules and budding rules are enabled in the same computation step, then one type of rules is nondeterministically chosen. When a neuron budding rule is applied, at this step the associated neuron is closed, and thus it cannot receive spikes. In the next step, the neurons obtained by budding will be open.

The configuration of the system is described by its topology structure, the number of spikes associated with each neuron, and the state of each neuron (open or closed). We emphasize that the system introduced here contains no spikes in the initial configuration. Using the rules as described above, one can define transitions among configurations. Any sequence of transitions starting in the initial configuration is called a computation. A computation halts if it reaches a configuration where all the neurons are open and no rule can be used.

In what follows, we give an example to make the application of budding rules transparent. Neither spiking nor forgetting rules are used.

An example. Let $\Pi_{1}$ be an SN P system with budding rules, whose initial topological structure (composed by a single neuron $\sigma_{1}$ ) is shown in the left hand side of Figure 2. Let $\Pi_{1}$ contain the following six budding rules:

a. $\lambda[]_{1} \rightarrow(1,2)[]_{2}$,

b. $(1,2)[]_{2} \rightarrow(3,2)[]_{3}$,

c. $(1,2)[]_{2} \rightarrow(2,4)[]_{4}$,

d. $(2,3)[]_{3} \rightarrow(3,5)[]_{5}$,

e. $(2,4)[]_{4} \rightarrow(4,6)[]_{6}$,

f. $(4,6)[]_{6} \rightarrow(6,3)[]_{3}$.

In the initial configuration, neuron $\sigma_{1}$ has no neighbourhood and only rule $a$. is enabled. The application of rule $a$. produces a new neuron $\sigma_{2}$ with a synapse $(1,2)$ connecting it with $\sigma_{1}$. Now both neurons $\sigma_{1}$ and $\sigma_{2}$ have a neighbourhood (each one being the neighbourhood of the other), since a synaptic connection exists between them. In this circumstance, rule $a$. is disabled while rules $b$. and $c$. are enabled and may be applied in parallel to neuron $\sigma_{2}$. When these two rules are applied two new neurons $\sigma_{3}$ and $\sigma_{4}$ are created, with the associated synapses $(3,2)$ and $(2,4)$. In the resulting configuration, rules $b$. and $c$. are disabled since now neuron $\sigma_{2}$ has three neighbours. At this step only rule $e$. can be applied to neuron $\sigma_{4}$, producing a new neuron $\sigma_{6}$ with a synaptic connection $(4,6)$. Note that at this step rule $d$. was not enabled as the synapse of neuron $\sigma_{3}$ is $(3,2)$, instead of $(2,3)$ as required by the rule. Now only rule $f$. is enabled, which creates only the synapse $(6,3)$ because neuron $\sigma_{3}$ already exists. From now on no rule is enabled, and thus the computation halts.

\section{SN P systems solving SAT}

Let us now consider the NP-complete decision problem SAT [8, p. 39]. The instances of SAT depend upon two parameters: the number $n$ of variables, and the 


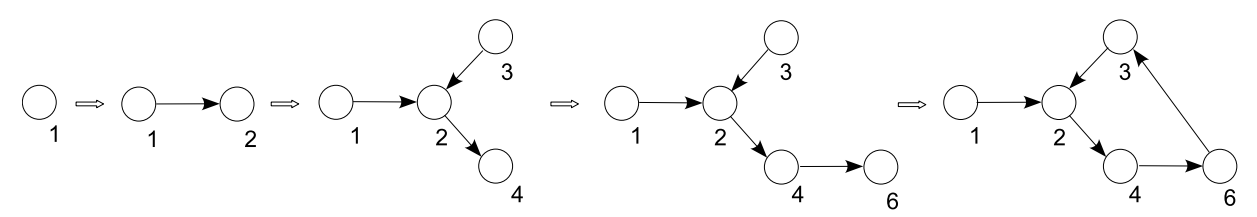

Fig. 2. Evolution of the structure of the SN P system $\Pi_{1}$, as the effect of the application of budding rules

number $m$ of clauses. We recall that a clause is a disjunction of literals, occurrences of $x_{i}$ or $\neg x_{i}$, built on a given set $X=\left\{x_{1}, x_{2}, \ldots, x_{n}\right\}$ of Boolean variables. Without loss of generality, we can avoid the clauses in which the same literal is repeated or both the literals $x_{i}$ and $\neg x_{i}$, for any $1 \leq i \leq n$, occur. In this way, a clause can be seen as a set of at most $n$ literals. An assignment of the variables $x_{1}, x_{2}, \ldots, x_{n}$ is a mapping $a: X \rightarrow\{0,1\}$ that associates to each variable a truth value. The number of all possible assignments to the variables of $X$ is $2^{n}$. We say that an assignment satisfies the clause $C$ if, assigned the truth values to all the variables which occur in $C$, the evaluation of $C$ (considered as a Boolean formula) gives 1 (true) as a result.

We can now formally state the SAT problem as follows.

Problem 1. NAME: SAT.

- Instance: a set $C=\left\{C_{1}, C_{2}, \ldots, C_{m}\right\}$ of clauses, built on a finite set $\left\{x_{1}, x_{2}\right.$, $\left.\ldots, x_{n}\right\}$ of Boolean variables.

- Question: is there an assignment to the variables $x_{1}, x_{2}, \ldots, x_{n}$ that satisfies all the clauses in $C$ ?

Equivalently, we can say that an instance of SAT is a propositional formula $\gamma_{n}=C_{1} \wedge C_{2} \wedge \cdots \wedge C_{m}$, expressed in the conjunctive normal form as a conjunction of $m$ clauses, where each clause is a disjunction of literals built using the Boolean variables $x_{1}, x_{2}, \ldots, x_{n}$. With a little abuse of notation, from now on we will denote by $\operatorname{SAT}(n, m)$ the set of instances of SAT which have $n$ variables and $m$ clauses.

In [6], a uniform family $\left\{\Pi_{S A T}(\langle n, m\rangle)\right\}_{n, m \in \mathbf{N}}$ of SN P systems was built such that for all $n, m \in \mathbb{N}$ the system $\Pi_{S A T}(\langle n, m\rangle)$ solves all the instances of $\operatorname{SAT}(n, m)$ in a number of steps which is quadratic in $n$ and linear in $m$. Here $\langle n, m\rangle$ denotes the natural number obtained by applying the Cantor bijection to the pair $(n, m)$ of natural numbers; so doing, the family of $\mathrm{P}$ systems depends upon one parameter instead of two. We assume that the reader is familiar with the construction given in [6]; for his convenience, here we summarize the structure and functioning of the system $\Pi_{S A T}(\langle n, m\rangle)$. In the next section, we are going to build such a system by means of budding rules.

Because the construction is uniform, we need a way to encode any given instance $\gamma_{n}$ of $\operatorname{SAT}(n, m)$. As stated above, each clause $C_{i}$ of $\gamma_{n}$ can be seen as a disjunction of at most $n$ literals, and thus for each $j \in\{1,2, \ldots, m\}$ either $x_{j}$ 


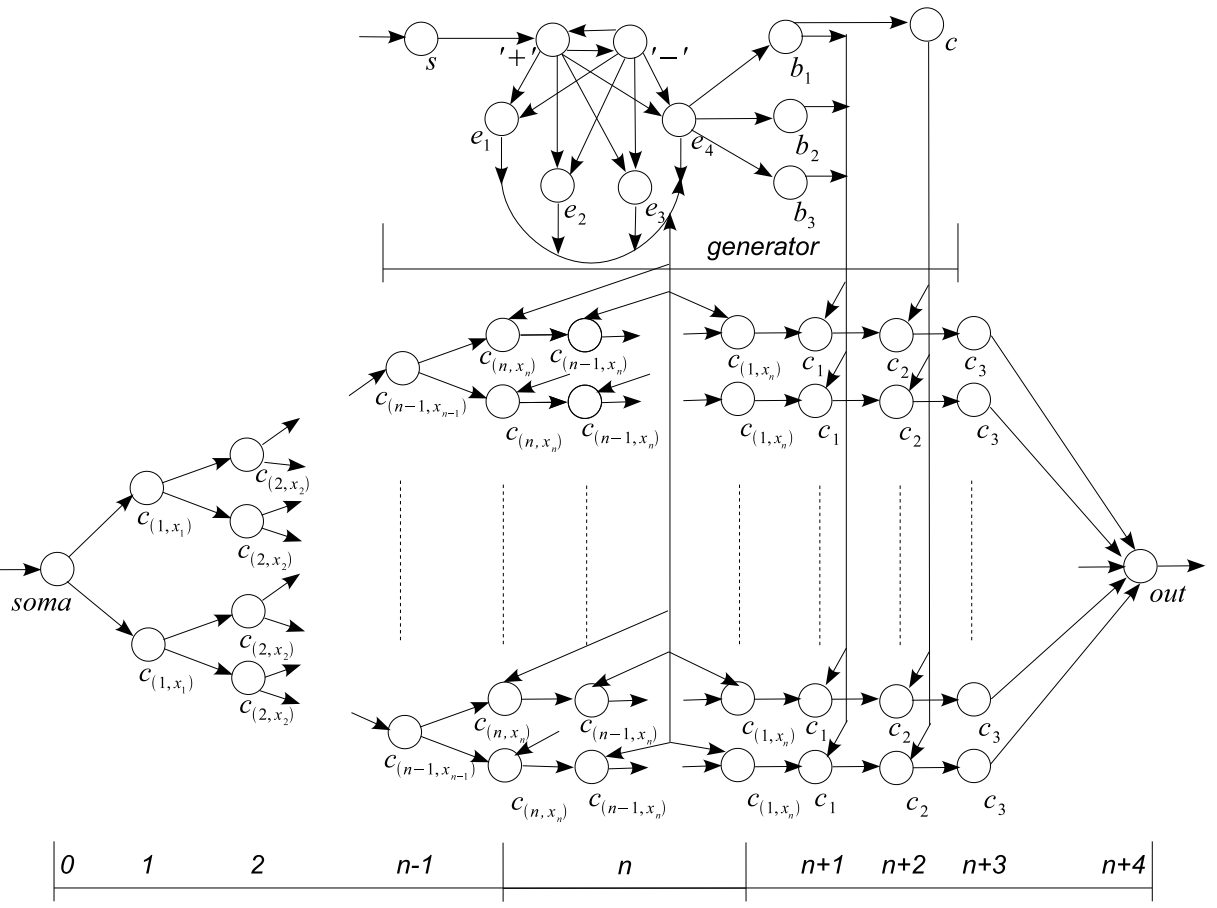

Fig. 3. A SN P system structure devoted to solve all the instances of $\operatorname{sAT}(n, m)$

occurs in $C_{i}$, or $\neg x_{j}$ occurs, or none of them occurs. In order to distinguish these three situations we define the spike variables $\alpha_{i j}$, for $1 \leq i \leq m$ and $1 \leq j \leq n$, as variables whose values are amounts of spikes, and we assign to them the following values:

$$
\alpha_{i j}= \begin{cases}a & \text { if } x_{j} \text { occurs in } C_{i} \\ a^{2} & \text { if } \neg x_{j} \text { occurs in } C_{i} \\ \lambda & \text { otherwise }\end{cases}
$$

So doing, clause $C_{i}$ will be represented by the sequence $\alpha_{i 1} \alpha_{i 2} \cdots \alpha_{i n}$ of spike variables; in order to represent the entire formula $\gamma_{n}$ we just concatenate the representations of the single clauses, thus obtaining the sequence $\alpha_{11} \alpha_{12} \cdots \alpha_{1 n} \alpha_{21} \alpha_{22} \cdots \alpha_{2 n} \cdots \alpha_{m 1} \alpha_{m 2} \cdots \alpha_{m n}$. As an example, the representation of $\gamma_{3}=\left(x_{1} \vee \neg x_{2}\right) \wedge\left(x_{1} \vee x_{3}\right)$ is the sequence $a a^{2} \lambda a \lambda a$.

The system structure is composed of $n+5$ layers, as illustrated in Figure 3. The first layer (numbered by 0 ) is used to insert into the system the representation of the instance of $\operatorname{sat}(n, m)$ to be solved, encoded as stated above. Note that each layer from 1 to $n$ contains two times the neurons contained in the previous layer. In this way we obtain in the $n$-th layer $2^{n}$ copies of a subsystem which is a sequence of $n$ neurons; each subsystem is bijectively associated to one of the 
possible assignments to the variables $x_{1}, x_{2}, \ldots, x_{n}$. The neurons that occur in each subsystem are of two types: $f$ and $t$. The type of a neuron indicates that the corresponding Boolean variable is assigned with the Boolean value $t$ (rue) or $f($ alse $)$, respectively. These subsystems, together with the so called generator, have a very specific function in the overall SN P system: to test (in parallel) all possible assignments against a given clause.

The assignment is performed by sending 3 spikes to all the neurons labelled with $t$, and 4 spikes to all the neurons labelled with $f$. This means that neurons $e$ in the generator will have three synapses going to neurons $t$ and four synapses towards neurons $f$. All these spikes arrive every $n$ computation steps, when the spikes indicated by the spike variables $\alpha_{i j}$ that correspond to a clause of $\gamma_{n}$ are contained into the subsystems of layer $n$. This process is started by putting one spike in neuron $s$ at the beginning of the computation. The delay associated with the rule contained in neuron $s$ allows to send the first spikes from neurons $e$ to neurons $t$ and $f$ exactly when the first clause is contained in layer $n$.

Recall our encoding of literals in the clauses (1): we have 0 spikes if the variable does not occur in the clause, 1 spike if it occurs non negated, and 2 spikes if it occurs negated. These spikes are added with those representing the assignments,

\begin{tabular}{|c|c|c|c|c|}
\hline & Assign. to $x_{j}$ & Literal & N. of spikes & Truth value of $C_{i}$ \\
\hline Neuron $t$ & $\begin{array}{l}\text { true } \\
\text { true } \\
\text { true }\end{array}$ & $\begin{array}{c}x_{j} \notin C_{i} \\
x_{j} \in C_{i} \\
\neg x_{j} \in C_{i}\end{array}$ & $\begin{array}{l}3+0=3 \\
3+1=4 \\
3+2=5\end{array}$ & $\begin{array}{l}? \\
\text { true } \\
?\end{array}$ \\
\hline Neuron $f$ & $\begin{array}{l}\text { false } \\
\text { false } \\
\text { false }\end{array}$ & $\begin{array}{c}x_{j} \notin C_{i} \\
x_{j} \in C_{i} \\
\neg x_{j} \in C_{i}\end{array}$ & $\begin{array}{l}4+0=4 \\
4+1=5 \\
4+2=6\end{array}$ & $\begin{array}{c}? \\
? \\
\text { true }\end{array}$ \\
\hline
\end{tabular}

Table 1. Number of spikes resulting from the assignment in the neurons of layer $n$, and its effect on the truth value of the clause

and the possible results are illustrated in Table 1. From this table we can see that if a neuron labelled with $t$ receives a total number of 4 spikes then the corresponding variable occurs non negated in the clause and is assigned the truth value true; we can immediately conclude that the clause is satisfied, and thus the neuron sends one spike towards the next layer. Similarly, if a neuron labelled with $f$ receives 6 spikes then the corresponding variable occurs negated in the clause and is assigned the truth value false; also in this case we can immediately conclude that the clause is satisfied, and the neuron signals this event by sending one spike towards the next layer. In all the other cases we cannot conclude anything on the truth value of the clause, and thus no spike is emitted. 
All the spikes which are emitted by neurons $t$ and $f$ are propagated through the neurons that compose layer $n$, until they reach the corresponding neuron $\sigma_{1}$ in layer $n+1$. Such a neuron is designed to make neuron $\sigma_{2}$ (in layer $n+2$ ) retain only one spike from those received by layer $n$. Hence, those assignments that satisfy the clause produce a single spike in the corresponding neuron $\sigma_{2}$; such a spike is accumulated in the associated neuron $\sigma_{3}$ (in layer $n+3$ ), that operates like a counter. When the first clause of $\gamma_{n}$ has been processed, the second enters into the system (in $n$ steps) and takes place in the subsystems; then all possible assignments are tested against this clause, and so on for all the clauses. When all the $m$ clauses of $\gamma_{n}$ have been processed, neurons $\sigma_{3}$ in layer $n+3$ contain each the number of clauses which are satisfied by the corresponding assignment. The neurons that contain $m$ spikes fire, sending one spike to neuron $\sigma_{\text {out }}$, thus signalling that their corresponding assignment satisfies all the clauses of the instance. Neuron $\sigma_{\text {out }}$ operates like an OR gate: it fires if and only if it contains at least one spike, that is, if and only if at least one of the assignments satisfies all the clauses of $\gamma_{n}$. Further technical details will be presented in the last part of the next section.

\section{A uniform solution to SAT by SN P systems with budding rules}

In this section we show that the pre-computed structures which are used in [6] to solve the instances of $\operatorname{SAT}(n, m)$ can be built in a polynomial time by SN P systems with budding rules. The SN P system with budding rules that we are going to define is composed of two subsystems: a first subsystem builds the structure of a second subsystem, that solves the instances of $\operatorname{SAT}(n, m)$ as described in the previous section. For the sake of simplicity, we avoid to use the neuron budding and the spiking rules at the same time in each subsystem.

Formally, the SN P system with budding rules is defined as

$$
\Pi=(O, \Sigma, H, \text { syn }, R, \text { soma }, \text { out })
$$

where:

1. $O=\{a\}$ is the singleton alphabet;

2. $\Sigma=\left\{\sigma_{i} \mid i \in H_{0}\right\}$ is the set of initial neurons;

3. $H$ is a finite set of labels for neurons, and $H \supseteq H_{0}=\left\{\right.$ soma, out, $\left.e_{0}, e_{1}, e_{2}, e_{3}, b_{1}, b_{2}, b_{3}, c, s,+,-\right\}$ is the set of labels for the neurons initially given;

4. syn $\subseteq H \times H$ is a finite set of synapses, with $(i, i) \notin$ syn for $i \in H)$, and syn $\supseteq \operatorname{syn}_{0}=\left\{\left(e, e_{i}\right) \mid 0 \leq i \leq 3, e \in\{+,-\}\right\} \cup\left\{\left(e_{0}, b_{i}\right) \mid 1 \leq i \leq 3\right\} \cup$ $\left\{\left(b_{3}, c\right),(s,+),(+,-),(-,+), \lambda\right\}$ is the set of synapses initially in use;

5. soma and out are the labels for the input and output neuron, respectively;

6. $R$ is a set of neuron budding and extended spiking rules defined as follows. 


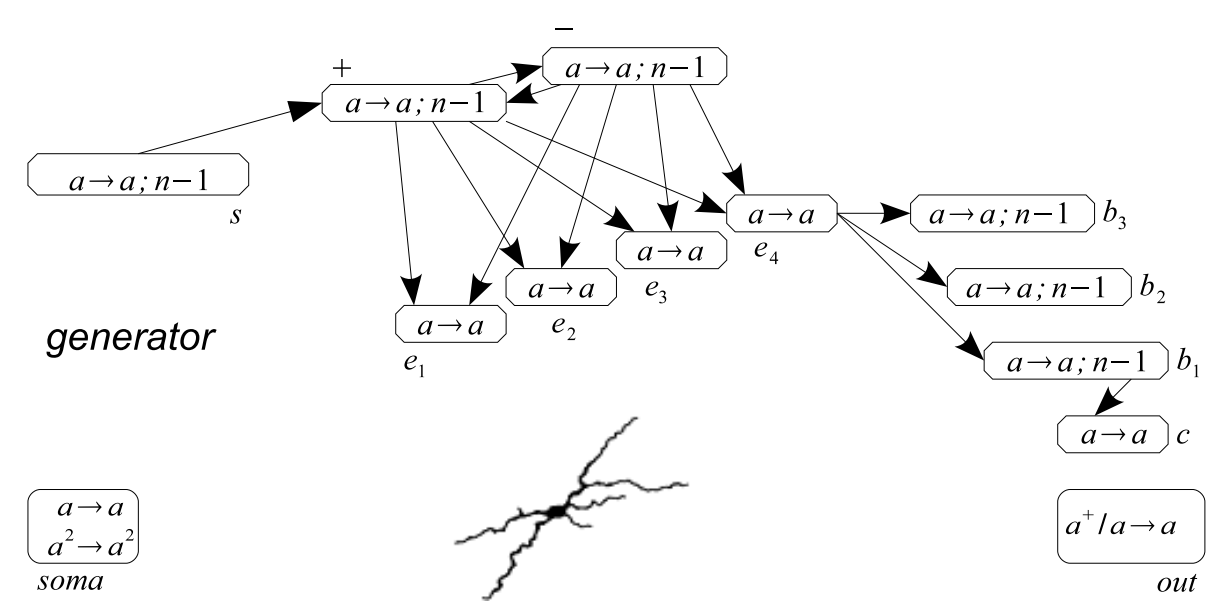

Fig. 4. The initial topological structure (newly born dendrite) of the SN P system $\Pi$ : the input (soma) and the output (out) neurons, and the generator

\section{Building the system structure.}

The system initially contains an input neuron $\sigma_{\text {soma }}$, an output neuron $\sigma_{\text {out }}$, and a sub-structure $G$ (named the generator) which is composed of the set of neurons specified in $\Sigma$ and the set of synapses from syn $n_{0}$, arranged as illustrated in Figure 4.

The generator is governed only by neuron budding rules, and is controlled by the labels of budding neurons and by the synapses created during the computation. The system construction algorithm consists of two phases:

A. Generation of the dendritic-tree sub-structure (the layers from 0 to $n$ in Figure 3 ) and assignment of the truth values to the $n$ Boolean variables. The process starts from the initial neuron $\sigma_{\text {soma }}$ (the root node) and produces $2^{n}$ neurons in $n$ steps. The label of each neuron in layer $n$ encodes an associated truth assignment.

B. Completion of the network structure. The neurons in the $n$-th layer of the system establish connections with the generator, according to the truth assignments represented in those neurons. The structure is then further expanded by three layers, and finally all the neurons in the last layer are connected with the output neuron $\sigma_{\text {out }}$.

Let us now describe in depth each of these phases.

Phase A. In this phase of computation, the dendritic tree (which is a complete binary tree) is generated in $n$ steps by applying budding rules of type $\mathbf{a}_{\mathbf{1}}$ ), described below, starting from an initial neuron $\sigma_{\text {soma }}$. The dendritic tree generation process is controlled by the labels of the neurons as well as by the synapses generated so far. 
It is worth to note that, since the truth assignments associated with the neurons in $n$-th layer are encoded in the labels of those neurons, also the truth assignments to the variables $x_{1}, x_{2}, \ldots, x_{n}$ are generated during the construction of the dendritic tree.

The label of a neuron $\sigma_{c}$ in layer $i$ is a sequence of the form

$$
c=\left(i, x_{i}^{(p)}\right)=\left(i, x_{i}(1)=p\right)=\left(i, p, x_{k 2}, \ldots, x_{i i}\right)
$$

with $p \in\{t, f\}$, where the first entry $(i)$ indicates the number of layer, while $x_{i}^{(p)}$ is a subsequence of length $i$ formed by the Boolean values $t$ and $f$ that have been generated up to now, that represents a truth assignment to the variables $x_{1}, x_{2}, \ldots, x_{i}$. The component $p$ in $x_{i}^{(p)}$ indicates that the first entry of the subsequence is exactly $p$.

An almost complete structure of the SN P system that solves the instances of $\operatorname{SAT}(2, m)$ is illustrated in Figure 5 . It is worth to follow its construction.

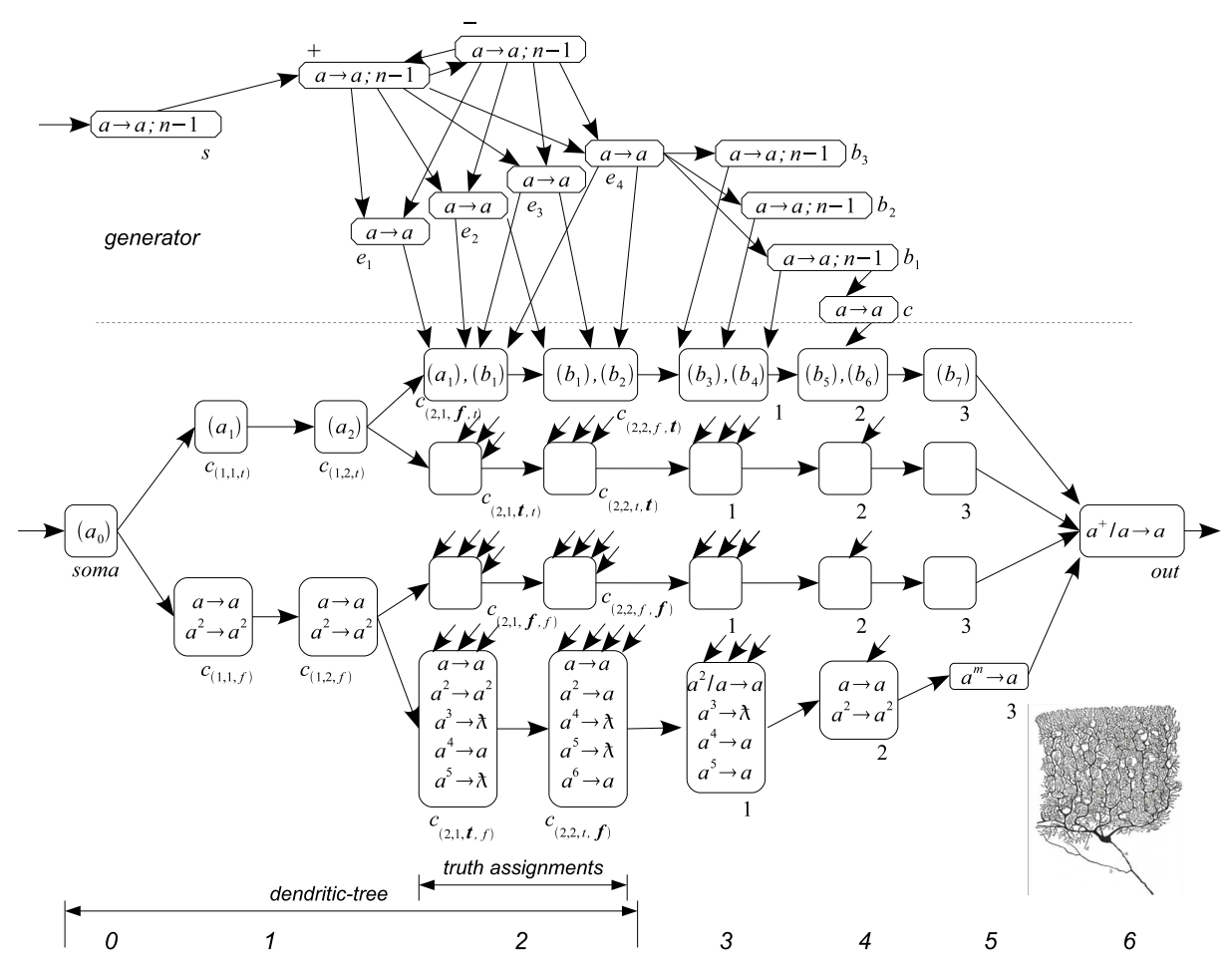

Fig. 5. An almost complete structure (maturated dendrite tree) of the $\mathrm{P}$ system for solving the instances of $\operatorname{SAT}(2, m)$. The neuron budding rules used in each computation step are indicated by their labels in the corresponding neurons. Some of the spiking rules are also indicated 
a1) $\left(c_{\left(i, x_{i-1}\right)}, c_{\left(i, x_{i}\right)}\right)[]_{c_{\left(i, x_{i}\right)}} \rightarrow\left(c_{\left(i, x_{i}\right)}, c_{\left(i+1, p, x_{i}\right)}\right)[]_{c_{\left(i+1, p, x_{i}\right)}}$,

$0 \leq i \leq n-1, p \in\{t, f\}, x_{i} \in\{t, f\}^{i},\left(c_{\left(-1, x_{-1}\right)}, c_{\left(0, x_{0}\right)}\right)=\lambda, c_{(0, \lambda)}=$ soma.

The computation starts by applying two rules of type $\mathbf{a}_{\mathbf{1}}$ ), for $i=0$, to the input neuron $\sigma_{c_{s o m a}}$. These two rules are:

$$
[]_{c_{\text {soma }}} \rightarrow\left(c_{\text {soma }}, c_{(1, t)}\right)[]_{c_{(1, t)}}, \quad \text { and } \quad[]_{c_{\text {soma }}} \rightarrow\left(c_{\text {soma }}, c_{(1, f)}\right)[]_{c_{(1, f)}},
$$

where $\left(c_{\text {soma }}, c_{(1, t)}\right),\left(c_{\text {soma }}, c_{(1, f)}\right) \in$ syn.

The left hand side of each rule (where $\lambda \in s^{s y n_{0}}$ is omitted) requires that its interaction environment be empty, i.e., no synapse exists connected to neuron $\sigma_{c_{s o m a}}$. As the left hand sides of both these rules are the same, and satisfy the constraints posed on the interaction environment of neuron $\sigma_{c_{s o m a}}$, they are applied simultaneously. As a result, two new neurons are budded: $\sigma_{c_{(1, t)}}$, with a synapse $\left(c_{\text {soma }}, c_{(1, t)}\right)$ coming from the father neuron, and $\sigma_{c_{(1, f)}}$, connected with the father neuron by a synapse $\left(c_{\text {soma }}, c_{(1, f)}\right)$. The symbols $t$ and $f$ in the neuron labels indicate the truth values true and false, respectively, and can be regarded as the two truth assignments $(t)$ and $(f)$ of length 1 for a single Boolean variable $x_{1}$. The first layer of the dendritic tree is thus established, and rules of type $\mathbf{a}_{\mathbf{1}}$ ) cannot be applied anymore, since the interaction environment of neuron $\sigma_{c_{s o m a}}$ has changed.

At the second computation step $(i=1)$, the following two rules are enabled and can be applied to each of the newly created neurons:

$$
\begin{aligned}
&\left(c_{\text {soma }}, c_{(1, t)}\right)[]_{c_{(1, t)}} \rightarrow\left(c_{(1, t)}, c_{(2, f, t)}\right)[]_{c_{(2, f, t)}}, \\
&\left(c_{\text {soma }}, c_{(1, t)}\right)[]_{c_{(1, t)}} \rightarrow\left(c_{(1, t)}, c_{(2, t, t)}\right)[]_{c_{(2, t, t)}}
\end{aligned}
$$

for $\sigma_{c_{(1, t)}}$, and

$$
\begin{aligned}
&\left(c_{\text {soma }}, c_{(1, f)}\right)[]_{c_{(1, f)}} \rightarrow\left(c_{(1, f)}, c_{(2, t, f)}\right)[]_{c_{(2, t, f)}}, \\
&\left(c_{\text {soma }}, c_{(1, f)}\right)[]_{c_{(1, f)}} \rightarrow\left(c_{(1, f)}, c_{(2, f, f)}\right)[]_{c_{(2, f, f)}}
\end{aligned}
$$

for $\sigma_{c_{(1, f)}}$. The former pair of rules yields to two new neurons having label $c_{(2, f, t)}$ and $c_{(2, t, t)}$, respectively; the synapses specified in these rules are budded from the neuron labelled with $c_{(1, t)}$. The latter pair of rules generates two neurons with labels $c_{(2, f, f)}$ and $c_{(2, t, f)}$, respectively; the synapses mentioned in these rules go from the neuron labelled with $c_{(1, f)}$ to the newly created neurons. In the meanwhile the truth assignments $(f, t),(t, t),(f, f),(t, f)$, for the Boolean variables $x_{1}$ and $x_{2}$, are generated at each leaf node, as illustrated in Figure 5. Since the interaction environment of neurons $\sigma_{c_{(1, t)}}$ and $\sigma_{c_{(1, f)}}$ has changed, the rules applied in this step cannot be applied anymore to these neurons.

By continuing in this way, by applying the budding rules of type $a_{1}$ ) in a maximally parallel way for $n$ computation steps, a complete binary tree of depth $n$ having $2^{n}$ leaves (hence an exponentially large workspace) is built. The label of each leaf node encodes a truth assignment of length $n$, hence all possible truth assignments for the Boolean variables $x_{1}, x_{2}, \ldots, x_{n}$ are generated. 
Phase B. The pre-computation to construct the SN P system structure continues until it converges to the output neuron in a further few steps. The main goal of this part of the construction algorithm is to design the substructure which is devoted to test the satisfiability of the clauses of the instance $\gamma_{n}$ of $\operatorname{SAT}(n, m)$ given as input against all possible truth assignments, and to determine whether there exist some assignments that satisfy all the clauses of $\gamma_{n}$.

The substructure is composed of $2^{n}$ subsystems, each being a sequence of $n$ neurons $\sigma_{c_{\left(j, x_{n}\right)}}, 1 \leq j \leq n$, including the leaf nodes of the dendritic tree. A subsequence $x_{n}=\left(x_{n 1}, x_{n 2}, \ldots, x_{n n}\right) \in\{t, f\}^{n}$ in a neuron label $c_{\left(j, x_{n}\right)}$ represents a truth assignment, and we can abstractly assign a pair $\left(j, x_{n}(j)\right)$ to a neuron $\sigma_{c_{\left(j, x_{n}\right)}}$ as its identity. Thus each subsystem represents a truth assignment formed by its neurons' identities. As stated above, a neuron with identification $\left(j_{1}, x\left(j_{1}\right)=t\right)$ has 3 synapses coming from the generator module, whereas a neuron with identity $\left(j_{2}, x\left(j_{2}\right)=f\right)$ is connected with the generator by means of 4 synapses. As we will see, these connections are used to perform assignments to the Boolean variables $x_{1}, x_{2}, \ldots, x_{n}$ that compose $\gamma_{n}$, and to check which assignments satisfy the clause of $\gamma_{n}$ currently under consideration.

For instance, the case in which $n=2$ is described in Figure 5 , where $2^{2}=4$ different truth assignments of length 2 have been generated for the two Boolean variables $x_{1}$ and $x_{2}$. The first subsystem is composed of two neurons having labels $c_{(2, f, t)}$ and $c_{(1, f, t)}$, respectively. The former is associated with the Boolean value false, as $x_{2}=(f, t)$ and $x_{2}(2)=f$, while the latter is associated with true, as $x_{2}(1)=t$; altogether they form the truth assignment $(f, t)$. The other subsystems are similar, and are associated with the truth assignments $(t, t),(f, f)$ and $(t, f)$. One can see that the four truth assignments are well distinguished from each other by the layer structure of the four subsystems.

To build the substructure of $n$ layers mentioned above, from now on two rules of types $\mathbf{a}_{2}$ ) and $\mathbf{a}_{\mathbf{3}}$ ) are applied simultaneously to a same neuron for $n-1$ steps. The first rule creates a new neuron with an associated synapse, while the second rule creates 3 or 4 synapses to the generator block. The same process occurs during the $n$-th step, by means of the rules of types $\mathbf{a}_{3}$ ) and $\mathbf{a}_{4}$ ); note that in this step the rules of type $\mathbf{a}_{2}$ ) cannot be applied anymore.

$\left.\mathbf{a}_{\mathbf{2}}\right)\left(c_{\left(n+1-j, x_{n}\right)}, c_{\left(n-j, x_{n}\right)}\right)[]_{c_{\left(n-j, x_{n}\right)}} \rightarrow\left(c_{\left(n-j, x_{n}\right)}, c_{\left(n-1-j, x_{n}\right)}\right)[]_{c_{\left(n-1-j, x_{n}\right)}}$, $p \in\{t, f\}, 0 \leq j \leq n-1,1 \leq k \leq n$,

$c_{\left(k, 0, x_{k}^{(p)}\right)}=c_{\left(k-1, n, x_{k-1}\right)}, x_{k}^{(p)}=\left(p, x_{k-1}\right) \in\{t, f\}^{k}$.

a $)\left(c_{\left(n+1-j, x_{n}\right)}, c_{\left(n-j, x_{n}\right)}\right)[]_{c_{\left(n-j, x_{n}(j+1)=p\right)}} \rightarrow\left(c_{\left(n-j, x_{n}(j+1)=p\right)}, e_{i}\right)[]_{e_{i}}$,

$0 \leq j \leq n, p \in\{t, f\}$ and $s \leq i \leq 3$, where $s=1$ if $p=t$, and $s=0$ if $p=f$,

$c_{\left(n, 0, x_{n}\right)}=c_{\left(n-1, n, x_{n}\right)}$.

We are now in the $(n+1)$-th step of the computation. When $j=0$, both rules of types $\left.\mathbf{a}_{2}\right)$ and $\left.\mathbf{a}_{3}\right)$ are applicable to each neuron $\sigma_{c_{\left(n, x_{n}\right)}}$ of layer $n$. The former rules generate neurons $\sigma_{c_{\left(n-1, x_{n}\right)}}$ with a synapse $\left(c_{\left(n, x_{n}\right)}, c_{\left(n-1, x_{n}\right)}\right)$. The latter type of rules creates three synapses to all neurons of type $\sigma_{c_{\left(n, x_{n}(1)=t\right)}}$ coming from the neurons $\sigma_{c_{e_{i}}}, 1 \leq i \leq 3$, and four synapses to the neurons 
$\sigma_{c_{\left(n, x_{n}(1)=f\right)}}$ coming from the four neurons $\sigma_{c_{e_{i}}}, 0 \leq i \leq 3$, of the generator block. The neuron budding rules of type $\mathbf{a}_{2}$ ) and the synapse creation rules of type $\mathbf{a}_{3}$ ) are applied simultaneously to the same neurons (leaf nodes) in layer $n$ in the following $n-1$ steps, since their interaction environments coincide. The effect of the application of these rules is the production of neurons having connections with the generator block.

So doing, $2^{n}$ subsystems, each one composed of a sequence of $n$ neurons, are generated starting from layer $n$. In each subsystem, every neuron corresponding to the Boolean value true $\left(x_{n}(j)=t\right)$ is connected with the generator block by means of three synapses, while the neurons that correspond to the Boolean value false $\left(x_{n}(j)=f\right)$ are connected with the generator block by four synapses.

From the $(2 n+1)$-th step of the computation on, no interaction environment of any neuron in the system allow to activate the rules of type $\mathbf{a}_{2}$ ). Hence these rules cannot be applied, but the computation continues with the next types of rules.

a4) $\left(c_{\left(2, x_{n}\right)}, c_{\left(1, x_{n}\right)}\right)[]_{c_{\left(1, x_{n}\right)}} \rightarrow\left(c_{\left(1, x_{n}\right)}, c_{1}\right)[]_{c_{1}}$.

The rules of type $\mathbf{a}_{\mathbf{4}}$ ) can be applied in parallel to the leaf nodes (neurons) of layer $n$; they produce the neurons $\sigma_{c_{1}}$ forming the $(n+1)$-th layer and, meanwhile, the rules of type $\mathbf{a}_{\mathbf{3}}$ ) create synapses from these neurons to the generator block.

a5) $\left(c_{\left(1, x_{n}\right)}, c_{1}\right)[]_{c_{1}} \rightarrow\left(c_{1}, c_{2}\right)[]_{c_{2}}$,

a $)\left(c_{\left(1, x_{n}\right)}, c_{1}\right)[]_{c_{1}} \rightarrow\left(b_{i}, c_{1}\right)[]_{b_{i}}, 1 \leq i \leq 3$.

While the rules of type $\mathbf{a}_{\mathbf{5}}$ ) are applied to the neurons $\sigma_{c_{1}}$ and bud neurons $\sigma_{c_{2}}$, the rules of type $\mathbf{a}_{\mathbf{6}}$ ) are also applied and create three synapses coming from the neurons $\sigma_{b_{i}}, 1 \leq i \leq 3$, to each neuron $\sigma_{c_{1}}$. In this way, layer $n+2$ is formed.

a $)\left(c_{1}, c_{2}\right)[]_{c_{2}} \rightarrow\left(c_{2}, c_{3}\right)[]_{c_{3}}$,

a8) $\left(c_{1}, c_{2}\right)[]_{c_{2}}^{c_{2}} \rightarrow\left(c, c_{2}\right)[]_{c}$.

The rules of types $\left.\mathbf{a}_{\mathbf{7}}\right)$ and $\left.\mathbf{a}_{\mathbf{8}}\right)$ apply simultaneously to every neuron $\sigma_{2}$ having a synapse $\left(c_{1}, c_{2}\right)$. As a result, a new neuron $\sigma_{c_{3}}$ is budded with a connection $\left(c, c_{2}\right)$ coming from neuron $\sigma_{c}$. All the neurons $\sigma_{c_{2}}$ in the same layer are subjected to the same effect, since the rules are applied in the maximally parallel way.

a9) $\left(c_{2}, c_{3}\right)[]_{c_{3}} \rightarrow\left(c_{3}\right.$, out $)[]_{\text {out }}$.

The pre-computation of the SN $\mathrm{P}$ system structure is completed by forming the connections from the neurons $\sigma_{c_{3}}$ to the output neuron $\sigma_{\text {out }}$, by means of the rules of type $\mathbf{a g}$ ). These rule are applied in the maximally parallel way to all the neurons in layer $n+3$.

Summarizing, phases A and B build an empty (that is, containing no spikes) structure of an SN P system, that can be used to solve all the instances of $\operatorname{SAT}(n, m)$ in a linear (with respect to $n$ ) number of computation steps. The size of the structure is exponential with respect to $n$. 
Solving SAT (Phase C).

Given an instance $\gamma_{n}$ of $\operatorname{SAT}(n, m)$, we first encode it as a sequence of spike variables, as explained in Section 3, equation (1). Then, the computation of the system may start. The sequence of spikes encoding $\gamma_{n}$ is introduced in the system, using neuron $\sigma_{\text {soma }}$. Let us see at what spiking rules are used to compute the solution, with a brief description for each.

$\left.\mathbf{c}_{1}\right)[a \rightarrow a]_{c_{\left(i, x_{i}\right)}} ;\left[a^{2} \rightarrow a^{2}\right]_{c_{\left(i, x_{i}\right)}}$ $0 \leq i \leq n, x_{i} \in\{t, f\}^{i}, c_{\left(0, x_{0}\right)}=\operatorname{soma}$

c $\left.\mathbf{2}_{2}\right)[a \rightarrow a ; n-1]_{s}$.

We insert 0,1 or 2 spikes into the system by rule $\mathbf{c}_{\mathbf{1}}$ ) using the input neuron $\sigma_{\text {soma }}$, according to the value of the spike variable $\alpha_{i j}$ we are considering in the representation of $\gamma_{n}$. In the meanwhile we insert a single spike $a$ into neuron $\sigma_{s}$, to fire once the rule $\mathbf{c}_{2}$ ), thus activating the generator block.

Each spike, encoding a spike variable inserted into the input neuron, is duplicated and transmitted to the next layer of neurons. This duplication is performed $n$ times, until $2^{n}$ replicated copies of the spike are placed in the leaf nodes (in layer $n$ ) of the dendritic tree.

c $\left.\mathbf{c}_{3}\right)[a \rightarrow a]_{e_{i}} ; 0 \leq i \leq 3$, $[a \rightarrow a ; n-1]_{+} ;[a \rightarrow a ; n-1]_{-}$.

These are the spiking rules of the generator block. Each $n$ steps, the generator provides 3 and 4 spikes, respectively, to the neurons of layer $n$ associated with the truth values $t$ and $f$. This is made in order to test the satisfiability of a clause which has propagated through the layers of the dendritic tree, by checking it against all possible truth assignments to the variables $x_{1}, x_{2}, \ldots, x_{n}$.

In another $n$ steps, the $2^{n}$ copies of the clause of $\gamma_{n}$ take place in the corresponding subsystems located in layers from $n+1$ to $2 n$, where the satisfiability of the clause against all possible truth assignments is tested. For this purpose, the spike-truth values $a^{4}$ and $a^{3}$ are assigned from the generator to the spike-variables of the clause, according to the truth assignments represented by the neurons that compose the subsystems. In fact, recall that in each subsystem every neuron corresponding to the Boolean value true $\left(x_{n}(j)=t\right)$ is connected with the generator block by means of three synapses, while the neurons that correspond to the Boolean value false $\left(x_{n}(j)=f\right)$ are connected with the generator by means of four synapses. The satisfiability is then checked by means of the rules of types $\mathbf{c}_{\mathbf{4}}$ ) and $\mathbf{c}_{\mathbf{5}}$ ) residing in the neurons.

$\left.\mathbf{c}_{4}\right)[a \rightarrow a]_{t_{t}} ;\left[a^{3} \rightarrow \lambda\right]_{t_{t}} ;\left[a^{2} \rightarrow a^{2}\right]_{t_{1}} ;$

$\left[a^{4} \rightarrow a\right]_{t_{t}} ;\left[a^{5} \rightarrow \lambda\right]_{t_{t}} ;\left[a^{2} \rightarrow a\right]_{t_{0}} ;$

$t_{t}=c_{\left(j, x_{n}(j)=t\right)}, 1 \leq j \leq n$,

$t_{1}=c_{\left(j, x_{n}(j)=t\right)}, 2 \leq j \leq n$,

$t_{0}=c_{\left(1, x_{n}(n)=t\right)}, x_{n} \in\{t, f\}^{n}$. 
These are the spiking rules that reside in the neurons of layer $n$, associated with the Boolean value true (in Figure $5 n=2, \sigma_{c_{(2, t, f)}}$ stands for false while $\sigma_{c_{(1, t, f)}}$ stands for true). The rules $a^{2} \rightarrow a^{2}, a^{2} \rightarrow a$, and $a \rightarrow a$ are used to transmit the spike variables $a, a^{2}$ along the subsystems. Once a clause $C_{i}$ is ready to be tested for satisfiability, each neuron associated with true contains either one spike $(a)$, two spikes $\left(a^{2}\right)$ or is empty $(\lambda)$. As a spike variable $a$ represents the occurrence of a Boolean variable $x_{j}$ in $C_{i}$, to which a true value $\left(a^{3}\right)$ sent by the generator is assigned, resulting in a yes answer $\left(a^{4}\right)$, then it passes to the neuron $\sigma_{c_{1}}$ along the subsystem as an indication that $C_{i}$ is satisfied by a truth assignment in which the Boolean variable $x_{j}$ is true. On the other hand, if the Boolean value true $\left(a^{3}\right)$ is assigned to a spike variable that represents the occurrence of $\neg x_{j}$ in $C_{i}\left(a^{2}\right)$ or the fact that $x_{j}$ does not occur in $C_{i}(\lambda)$, then in these cases the answer is no, which is computed by the rules $a^{3} \rightarrow \lambda$ and $a^{5} \rightarrow \lambda$.

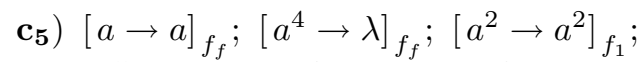

$\left[a^{5} \rightarrow \lambda\right]_{f_{f}} ;\left[a^{6} \rightarrow a\right]_{f_{f}} ;\left[a^{2} \rightarrow a\right]_{f_{0}} ;$

$f_{f}=c_{\left(j, x_{n}(j)=f\right)}, 1 \leq j \leq n$,

$f_{1}=c_{\left(j, x_{n}(j)=f\right)}, 2 \leq j \leq n$,

$f_{0}=c_{\left(1, x_{n}(n)=f\right)}, x_{n} \in\{t, f\}^{n}$.

These are the spiking rules that reside in the neurons of layer $n$, associated with the Boolean value false. The functioning of these rules is similar to that of rules $\left.\mathbf{c}_{4}\right)$.

c. $[a \rightarrow a ; n-1]_{b_{i}} ; 1 \leq i \leq 3$,

$\left[a^{2} / a \rightarrow a\right]_{c_{1}} ;\left[a^{3} \rightarrow \lambda\right]_{c_{1}} ;$

$\left[a^{4} \rightarrow a\right]_{c_{1}} ;\left[a^{5} \rightarrow a\right]_{c_{1}}$.

Whether an assignment satisfies or not the clause under consideration, is checked by a combined functioning of the neurons with label 1 in layer $n+1$ and the neurons with label $b_{i}, 1 \leq i \leq 3$, in the generator.

c $\begin{aligned} & {[a \rightarrow \lambda]_{c_{2}} ;\left[a^{2} \rightarrow a\right]_{c_{2}} ; } \\ & {[a \rightarrow a]_{c} . }\end{aligned}$

With a combined action of neuron $\sigma_{c}$, neuron $\sigma_{c_{2}}$ sends a spike to neuron $\sigma_{c_{3}}$ if and only if the corresponding assignment satisfies the clause under consideration.

c8) $\left[a^{m} \rightarrow a\right]_{c_{3}}$;

$\left[a^{+} / a \rightarrow a\right]_{\text {out }}$.

Neurons with label $c_{3}$ count how many clauses of the instance $\gamma_{n}$ are satisfied by the corresponding truth assignments. If one of these neurons get $m$ spikes, then it fires. Hence the number of spikes that reach neuron out is the number of assignments that satisfy all the clauses of $\gamma_{n}$. The output neuron fires if it contains at least one spike, thus signalling that the problem has a positive solution; otherwise, there is no assignment that satisfies the instance $\gamma_{n}$. 
This stage of computation ends at the $(n m+n+4)$-th step. The entire computation of the system thus halts in at most $n m+n+5$ computation steps, hence in a polynomial time with respect to $n$ and $m$.

In conclusion, we obtained a deterministic, polynomial time and uniform solution to $\operatorname{SAT}(n, m)$ in the framework of SN P systems.

\section{Conclusions and directions for future research}

In the present paper we proposed a way to solve the NP-complete decision problem SAT in a polynomial time with respect to the number $n$ of Boolean variables and the number $m$ of clauses that compose the instances of SAT being solved. Specifically, we introduced SN P systems with neuron budding rules, a new feature that enhances the efficiency of SN P systems by allowing them to generate an exponential size synapse graph (regarded as the workspace of the system) in a polynomial time with respect to $n$.

Neuron budding rules drive the mechanism of neuron production and synapse creation, according to the interaction of neurons with their neighbourhoods (described by the synapses that connect them to other neurons). We have shown that a very restricted type of neuron budding rules, involving one or two synapses (actually, when two synapses are involved, they appear one in each side of the rule) is sufficient to solve the SAT problem. The solution is computed in two stages: the first phase builds an exponential size SN P system that contains no spikes; then, this SN P system is fed with the instance of SAT to be solved (encoded in an appropriate way) and the answer is computed. The system works in the deterministic and maximally parallel manner.

The idea of producing new neurons in SN P systems is not new: already in [15] neurons are generated by division. However, both biological motivation and mathematical formal definition are different: neuron budding in this paper depends on the connections (structure) with other neurons, while neuron division depends on the number of spikes occurring inside the neurons (that is, the contents); hence they are two different ways to increase the workspace of SN P systems.

An open question is whether SN P systems with budding rules can be used to efficiently solve other computationally difficult problems, such as numerical NPcomplete problems and PSPACE-complete problems.

SN P systems with neuron budding rules can be extended by introducing more general rules, which in some sense capture the dynamic interaction of neurons with their neighbourhood. One possible form of such general rules is as follows: $A_{i}[]_{i} B_{i} \rightarrow C_{j}[]_{j} D_{j}$, where $A_{i}, B_{i}$ and $C_{j}, D_{j}$ are the sets of synapses coming to and going out from, respectively, the specified neurons $\sigma_{i}$ and $\sigma_{j}$. Clearly, in such general rules, more than one synapses can be involved in the neighbourhood of the considered neuron. 


\section{Acknowledgments}

The work of Tseren-Onolt Ishdorj was supported by BIOTARGET, a joint project between the University of Turku and Åbo Akademi University, funded by the Academy of Finland. The work of L. Pan was supported by the National Natural Science Foundation of China (Grant Nos. 60674106, 30870826, 60703047, and 60533010), Program for New Century Excellent Talents in University (NCET-05-0612), Ph.D. Programs Foundation of Ministry of Education of China (20060487014), Chenguang Program of Wuhan (200750731262), HUSTSRF (2007Z015A), and by the Natural Science Foundation of Hubei Province (2008CDB113 and 2008CDB180). The work of Alberto Leporati was partially supported by MIUR project "Mathematical aspects and emerging applications of automata and formal languages" (2007).

\section{References}

1. H. Chen, R. Freund, M. Ionescu, Gh. Păun, M.J. Pérez-Jiménez: On string languages generated by spiking neural P systems. Fundamenta Informaticae 75:141-162, 2007.

2. H. Chen, M. Ionescu, T.-O. Ishdorj: On the efficiency of spiking neural P systems. Proceedings of the $8^{\text {th }}$ International Conference on Electronics, Information, and Communication, Ulanbator, Mongolia, June 2006, pp. 49-52.

3. H. Chen, M. Ionescu, T.-O. Ishdorj, A. Păun, Gh. Păun, M.J. Pérez-Jiménez: Spiking neural P systems with extended rules. Fourth Brainstorming Week on Membrane Computing (M.A. Gutiérrez-Naranjo, Gh. Păun, A. Riscos-Núñez, F.J. RomeroCampero, eds.), vol. I, RGNC Report 02/2006, Research Group on Natural Computing, Sevilla University, Fénix Editora, 2006, pp. 241-266.

4. W. Gerstner, W. Kistler: Spiking neuron models. Single neurons, populations, plasticity. Cambridge University Press, 2002.

5. M. Ionescu, Gh. Păun, T. Yokomori: Spiking neural P systems. Fundamenta Informaticae 71(2-3):279-308, 2006.

6. T.-O. Ishdorj, A. Leporati: Uniform solutions to SAT and 3-SAT by spiking neural P systems with pre-computed resources. Natural Computing 7(4):519-534, 2008.

7. T.-O. Ishdorj, A. Leporati, L. Pan, X. Zeng, X. Zhang: Deterministic solutions to QSAT and Q3SAT by spiking neural P systems with pre-computed resources. Submitted for publication.

8. M.R. Garey, D.S. Johnson: Computers and intractability. A guide to the theory on NP-completeness. W.H. Freeman and Company, 1979.

9. A. Leporati, M.A. Gutiérrez-Naranjo: Solving SubSET Sum by spiking neural P systems with pre-computed resources. Fundamenta Informaticae 87(1):61-77, 2008.

10. A. Leporati, G. Mauri, C. Zandron, Gh. Păun, M. J. Pérez-Jiménez: Uniform solutions to SAT and SUBSET SUM by spiking neural P systems. Natural Computing (Online version), DOI: 10.1007/s11047-008-9091-y.

11. A. Leporati, C. Zandron, C. Ferretti, G. Mauri: Solving numerical NP-complete problem with spiking neural P systems. In: G. Elefterakis et al. (Eds.), Membrane Computing, $8^{\text {th }}$ International Workshop (WMC 8), Revised Selected and Invited Papers. LNCS 4860, Springer, 2007, pp. 336-352. 
12. A. Leporati, C. Zandron, C. Ferretti, G. Mauri: On the computational power of spiking neural P systems. International Journal of Unconventional Computing 5(5):459473, 2009.

13. W. Maass: Computing with spikes. Special Issue on Foundations of Information Processing of TELEMATIK 8(1):32-36, 2002.

14. W. Maass, C. Bishop (eds.): Pulsed neural networks. MIT Press, 1999.

15. L. Pan, Gh. Păun, M. J. Pérez-Jiménez: Spiking neural P systems with neuron division and budding. Seventh Brainstormin Week on Membrane Computing, (R. Gutiérrez-Escudero, M.A. Gutiérrez-Naranjo, Gh. Păun, I. Pérez-Hurtado, A. RiscosNúñez, eds.), vol. II, RGNC Report 01/2009, Research Group on Natural Computing, Sevilla University, Fénix Editora, 2009, pp. 151-167.

16. Gh. Păun: Membrane computing - An introduction. Springer-Verlag, Berlin, 2002.

17. Gh. Păun: Twenty six research topics about spiking neural P systems. Fifth Brainstorming Week on Membrane Computing (M.A. Gutiérrez-Naranjo, Gh. Păun, A. Romero-Jiménez, A. Riscos-Núñez, eds.), RGNC Report 01/2007, Research Group on Natural Computing, Sevilla University, Fénix Editora, 2007, pp. 263-280.

18. M. Sipser: Introduction to the theory of computation. PWS Publishing Company, Boston, 1997.

19. The P systems Web page: http://ppage.psystems.eu/

20. Think and Grow Toys: http://www.tagtoys.com/dendrites.php 